\title{
Helioseismic Observations of Solar Convection Zone Dynamics
}

\author{
Frank Hill, Rachel Howe, Rudi Komm, Irene González Hernández, \\ Shukur Kholikov, and John Leibacher
}

National Solar Observatory, Tucson, Arizona, USA 85726

\begin{abstract}
The large-scale dynamics of the solar convection zone have been inferred using both global and local helioseismology applied to data from the Global Oscillation Network Group (GONG) and the Michelson Doppler Imager (MDI) on board SOHO. The global analysis has revealed temporal variations of the "torsional oscillation" zonal flow as a function of depth, which may be related to the properties of the solar cycle. The horizontal flow field as a function of heliographic position and depth can be derived from ring diagrams, and shows near-surface meridional flows that change over the activity cycle. Time-distance techniques can be used to infer the deep meridional flow, which is important for flux-transport dynamo models. Temporal variations of the vorticity can be used to investigate the production of flare activity. This paper summarizes the state of our knowledge in these areas.
\end{abstract}

Keywords. Sun: helioseismology, Sun: interior, Sun: activity

\section{Introduction}

Since the advent of helioseismology in 1961, substantial advances have been made in our understanding of the large-scale dynamics in the solar convection zone. The modern era of helioseismology started in 1995 with the deployment of the Global Oscillation Network Group (GONG) set of ground-based instruments and the launch of the Solar and Heliospheric Observatory (SOHO) carrying three helioseismology instruments (MDI, GOLF and VIRGO). Now, with 15 years of data we can observe a number of features of the flows in the solar convection zone. Here we will briefly describe some of the latest observations pertaining to the torsional oscillation and solar cycle timing. We will also discuss deep and shallow meridional flows and divergence observations. Finally the relationship between vorticity, active regions and flares will be briefly reviewed.

\section{Torsional Oscillation and Solar Cycle Timing}

Howard \& LaBonte (1980) discovered a plasma stream on the solar surface that was slightly faster than the average surface rotation rate and that migrated from the solar poles to the equator over the course of the solar cycle. They named this east-west zonal flow the "torsional oscillation" since it had some of the characteristics of the process postulated by Walén (1944) as the basis of the solar cycle. Solar activity forms at the high-latitude boundary of the equatorward torsional oscillation stream, suggesting a close link between the flow and the activity. Long-term observations from Mt. Wilson show asymmetric patterns of the flow in the north and south solar hemispheres (Ulrich \& Boyden 2005). A similar pattern is observed in coronal emissions (Altrock et al. 2008).

Helioseismology has revealed that the torsional oscillation is not confined to the solar surface, but extends downward to depths of at least $60 \mathrm{Mm}$, half way through the solar convection zone (Howe et al. 2000, Vorontsov et al. 2002). It also shows that there is 


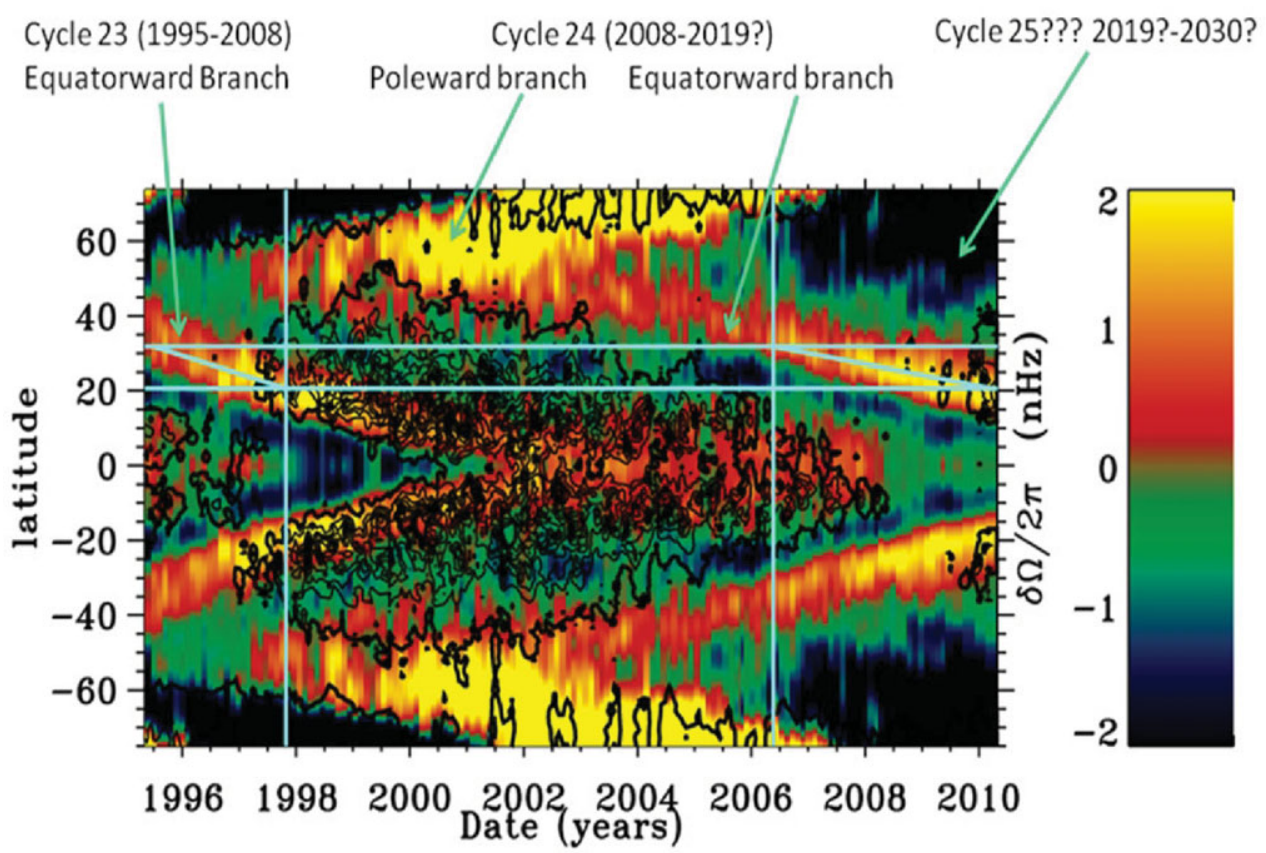

Figure 1. Torsional oscillation at a depth of $7 \mathrm{Mm}$ as a function of latitude and date. The magnetic field is overlaid as contours of $5 \mathrm{G}$. The labels at the top indicate the flows associated with cycles 23 and 24 , and where the first sign of cycle 25 , which is apparently late, should appear.

a variation in the solar rotation rate near the poles that can be interpreted as either a poleward branch of the torsional oscillation or as an alternating polar acceleration and deceleration. Fig. 1 shows the pattern of the torsional oscillation at a depth of 7 $\mathrm{Mm}$ as a function of latitude and date determined from global inversion of GONG and $\mathrm{SOHO} / \mathrm{MDI}$ data, with a contour overlay of the surface magnetic field.

It now appears that the timing of the solar cycle is well-correlated with the temporal evolution of the torsional oscillation (Howe et al. 2009). For example, the rate of migration of the equatorward branch is apparently related to the length of the minimum. Fig. 1 demonstrates that, for cycle 24, the equatorward migration took 1.5 years longer to reach a latitude of $20-25^{\circ}$, the point at which the activity ramped up in cycle 23 . The recent minimum was also about 1.5 years longer. Fig. 1 also shows that, when the equatorward branch reached the $20-25^{\circ}$ latitude range for cycle 23 , the surface activity increased rapidly. The same behavior is now being seen for cycle 24. Howe et al. (2009) pointed out that the position of the flow belts by mid-2009 corresponded to that at the epoch in the previous cycle when solar activity was just about to start rising - and indeed, by the end of 2009 the new cycle was finally underway. The equatorward branch appears several years prior to the surface magnetic field. For cycle 24, the equatorward branch appeared at the end of 2002, some 7 years prior to the activity. It is thus an early indication of the cycle timing. Finally, the poleward branch appears even earlier, about when the surface activity for the prior cycle increases rapidly. This was approximately 12 years in the case of cycle 24 . Note that the cycle 25 poleward branch has not yet appeared. Does this mean that cycle 25 will be very weak or even non-existent? Or is it simply delayed? 

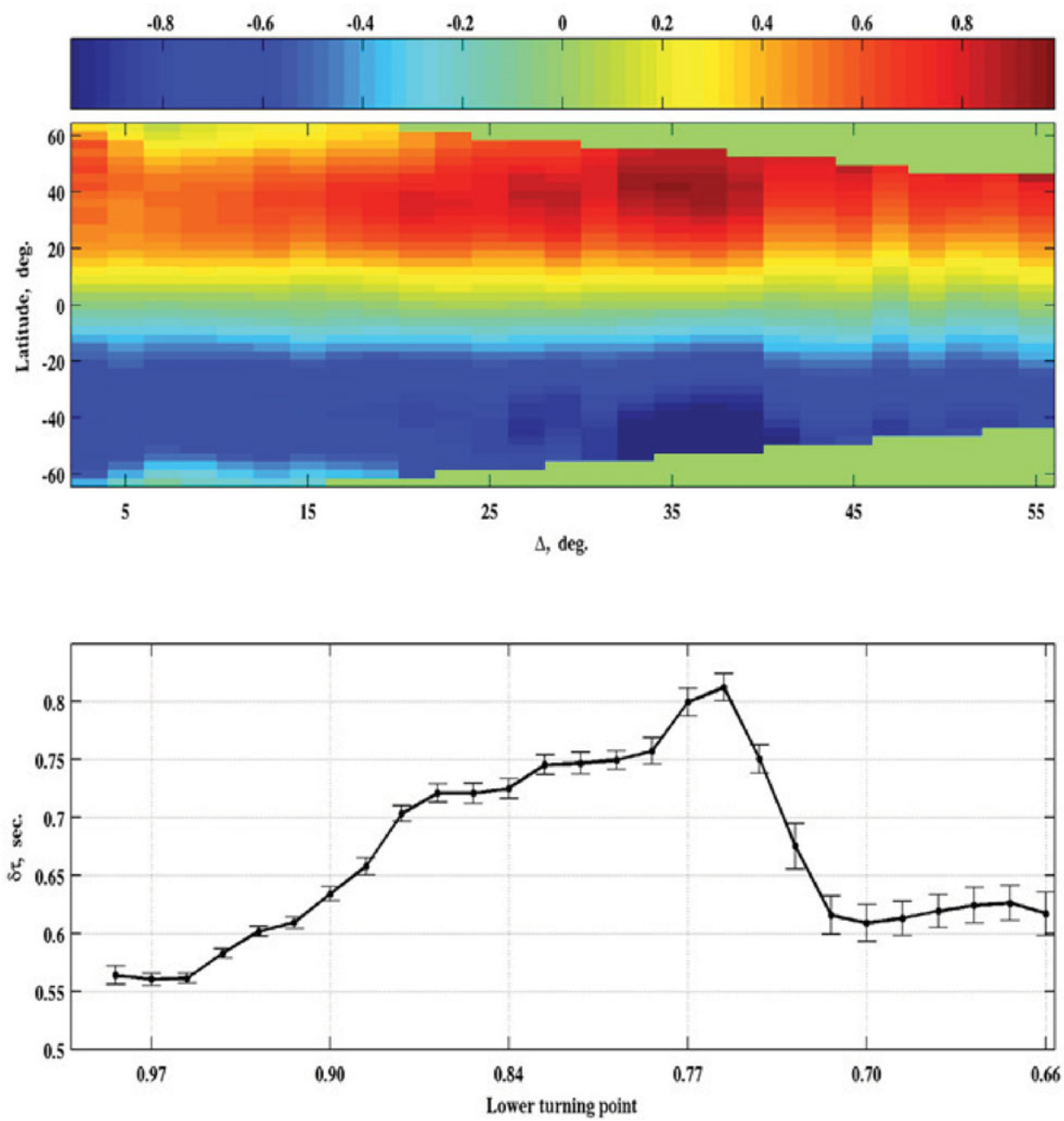

Figure 2. Deep meridional flow travel-time differences. Top: Average of 15 years of GONG meridional flow measurements as a function of the separation between the two latitudes that are correlated. The lower turning point for waves with travel distance of about $45^{\circ}$ reaches the tachocline region. Bottom: mean time difference averaged over both hemispheres for the latitude range $20^{\circ}-40^{\circ}$ as a function of propagation depth. The time difference increases until just before the bottom of the convective zone, then it sharply drops.

\section{Meridional Flows and Divergence}

According to flux-transport dynamo models, the north-south meridional flow plays an important role in determining the characteristics of the solar cycle (Dikpati \& Gilman 2009). In particular, the magnitude of the deep return flow (thought to be located at the base of the convection zone) from the pole to the equator sets the strength and timing of the activity. From estimates of the signal-to-noise ratio for measurements focused near the base of the convection zone, Braun \& Birch (2008) concluded that helioseismic measurements needed to span a solar cycle in order to detect the $1-2 \mathrm{~m} / \mathrm{s}$ return flow required by mass conservation. Fig. 2 shows the travel-time differences for 15 years of GONG data derived from near-sectoral modes that are primarily sensitive to the meridional flow. There is a distinct and significant change in the nature of the differences at 


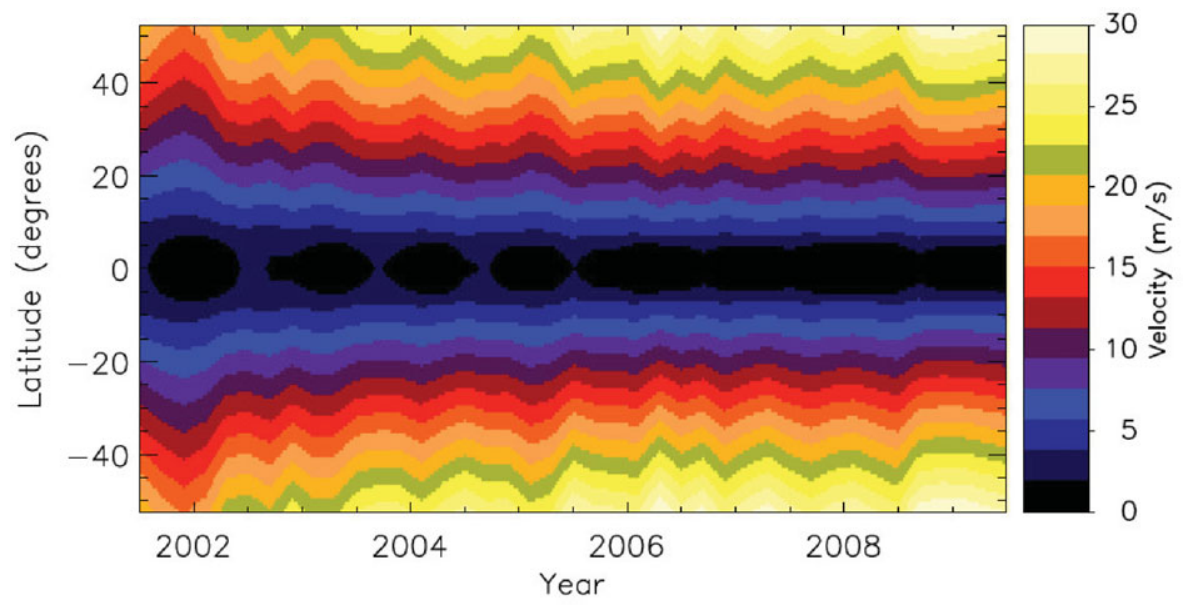

Figure 3. Temporal variation of a fitted polynomial to meridional circulation observations inferred at a depth of $5.8 \mathrm{Mm}$. Positive velocities are taken towards each respective pole.

the bottom of the convection zone, corresponding to a jump of about $2 \mathrm{~m} / \mathrm{s}$ with errors of about $0.2 \mathrm{~m} / \mathrm{s}$ (Leibacher, Kholikov \& Hill 2010). This indicates that these measurements have adequate signal-to-noise to resolve the predicted return flow amplitude. Determining the actual flow requires an inversion to cancel out near-surface effects. Preliminary inversions surprisingly indicate that there are multiple meridional cells in depth, and that the return flow may actually lie in the radiative envelope below the base of the convection zone. More work must be done to verify these results.

The meridional flow immediately below the surface contributes to the evolution of activity by transporting the surface magnetic field to the poles, canceling and reversing the polarity of the polar field. Since the strength of the polar field is correlated with the maximum level of activity in the subsequent cycle, variations in the near-surface meridional flow also play a role in setting the characteristics of the cycle. Fig. 3 shows that the near-surface meridional flow has been increasing in magnitude over the declining phase of cycle 23 (González Hernández et al. 2010). Hathaway \& Rightmire (2010) postulate that this increase of speed is one of the factors contributing to the peculiar minimum between cycles 23 and 24, and that cycle 24 will turn out to be a weak one.

The ring-diagram analysis of local helioseismology provides the horizontal flow field as a function of depth and heliographic position. Using the continuity equation and assuming incompressibility on large spatial scales allows us to derive the vertical velocity field, the divergence, and the vorticity (Komm 2007). Fig. 4 shows the divergence near the surface as a function of depth and latitude during maximum and minimum activity. The pattern of divergence indicates the presence of multiple cells in the north-south meridional direction, with a convergence at the active latitudes. The pattern, though substantially weaker, is also present at solar minimum.

It thus seems that there is considerable structure in the solar meridional flow in the form of multiple cells in both latitude and depth, and it is likely that the characteristics of these structures will vary in time. These aspects of the solar dynamics must be incorporated into dynamo models if the models are to explain the solar cycle. Indeed, recent simulations (e.g. Muñoz-Jaramillo, Nandy, \& Martens 2009, Dikpati et al. 2010) are beginning to include these new results into the calculations. 

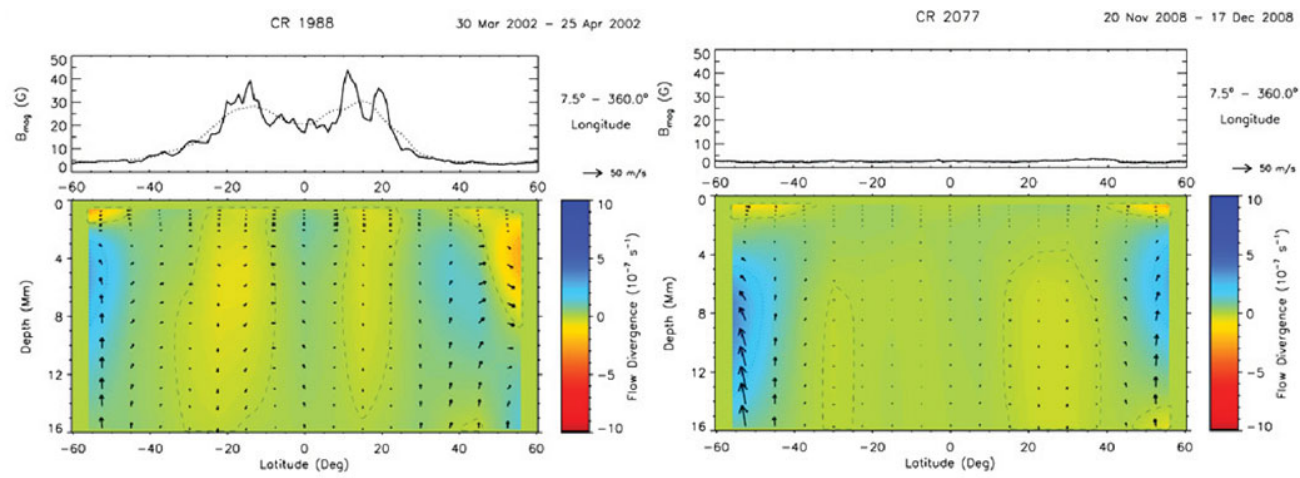

Figure 4. The divergence of flows as a function of depth and latitude for maximum (left) and minimum (right) activity levels. A multi-cellular pattern in latitude is visible that is more prominent during maximum.

\section{Subsurface Vorticity, Active Regions and Flares}

As stated, the ring diagram method can provide measurements of the vorticity below the photosphere. Mason et al. (2006) found that there is a specific pattern of vorticity below virtually every active region in the declining phase of cycle 23. Fig. 5 shows the pattern, which appears as four regions of oppositely directed horizontal vorticity. These can be interpreted as cross-sections of two vortex rings stacked on top of each other, with oppositely directed circulation in each ring. This is consistent with a downdraft from the solar surface caused by the cooling effect of the magnetic field meeting an upflow at a depth of about $10 \mathrm{Mm}$.

Further work found a correlation between the flaring activity in an active region, the strength of the subsurface vorticity, and the magnitude of the surface magnetic field (Komm \& Hill 2009). Fig. 6 shows that active regions with both strong vorticity and strong surface field are very likely to produce many strong flares. This suggests that the routine measurement of vorticity below active regions would provide a useful space weather forecast tool. In addition, the temporal variation of the subsurface vorticity has been demonstrated to be able to predict the occurrence of flares as much as three days in advance (Reinard et al. 2010). The physical mechanism is that the kinetic energy contained in the vortex rings is transformed into a twisting of the magnetic field that eventually overcomes the repulsion of like polarity and explosively reconnects.

\section{Conclusion}

Helioseismology has now matured to the point that it is revealing a number of new aspects of the relationship between internal solar dynamics and surface activity. The characteristics of the torsional oscillation, the meridional flow, and the vorticity below active regions seem to play major roles in determining the solar cycle and the evolution of the surface magnetic field on both long and short time scales. It thus seems that models of the surface activity must incorporate the sub-surface flow fields in order to accurately capture the evolution of the magnetic field in the photosphere and the corona.

\section{Acknowledgments}

This work was partially supported by NASA GIP Grant \#NNG08EI54I and NASA NNH07AG21I. This work utilizes data obtained by the Global Oscillation Network Group 


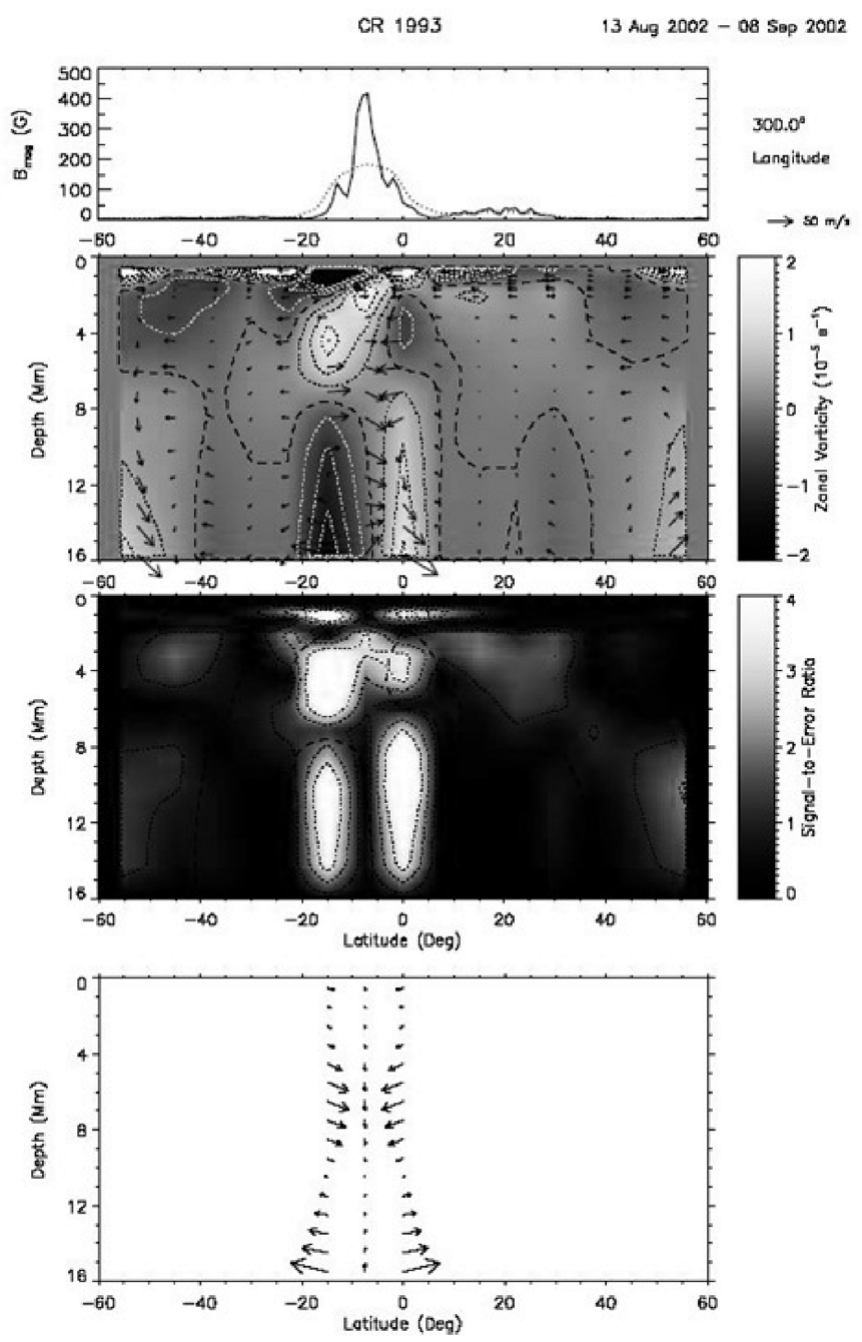

Figure 5. Zonal vorticity at $300^{\circ}$ longitude in CR 1993 as a function of latitude and depth. Top: Gross magnetic flux (solid line) and binned over $15^{\circ}$ (dotted line). Second panel: the $x$-component of vorticity. The arrows represent the meridional and vertical velocity components with the vertical magnitude increased by a factor of ten for visibility. Third panel: The signal-to-error ratio. Bottom panel: Idealized schematic of flows below a strong active region (with arbitrary amplitudes). From Mason et al. (2006), reproduced by permission of the AAS.)

(GONG) project, managed by the National Solar Observatory, a division of the National Optical Astronomy Observatories, which are operated by AURA, Inc., under a cooperative agreement with the National Science Foundation. The data were acquired by instruments operated by the Big Bear Solar Observatory, High Altitude Observatory, Learmonth Solar Observatory, Udaipur Solar Observatory, Instituto de Astrofísica de Canarias, and Cerro Tololo Inter-American Observatory.

On a personal note, since this Symposium was in honor of Juri Toomre, FH would like to thank Juri for all of the support and guidance he has provided to himself and to the GONG project over the years. 


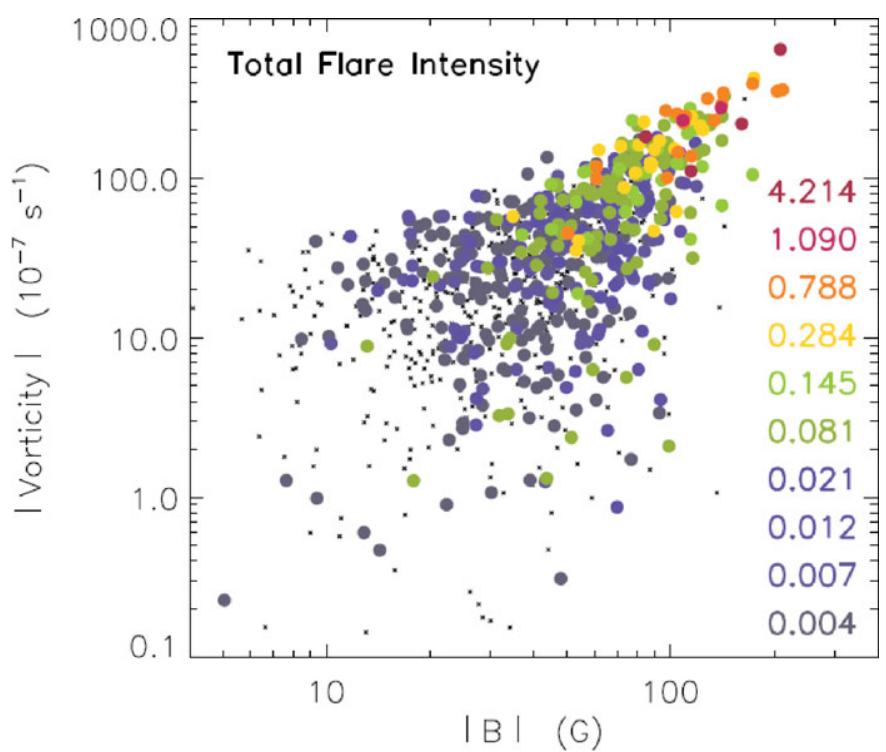

Figure 6. The location of approximately 1000 active regions from 2001 to 2006 in a (vorticity, surface magnetic field) plane. The size and gray scale of the points indicate the total flare productivity in units. The most flare-productive active regions have the highest values of both vorticity and surface magnetic field strength. From Komm \& Hill (2009), reproduced by permission of the AGU.

\section{References}

Altrock, R., Howe, R., \& Ulrich, R. K. 2008, in: R. Howe, R. W. Komm, K. S. Balasubramaniam \& G. J. D. Petrie (eds.), Subsurface and Atmospheric Influences on Solar Activity (San Francisco: Astronomical Society of the Pacific), p. 335

Braun, D. C. \& Birch, A. C. 2008, ApJ (Letters), 689, L161

Dikpati, M. \& Gilman, P. A. 2009, Space Sci. Revs, 144, 67

Dikpati, M., Gilman, P. A., de Toma, G., \& Ulrich, R. K. 2010, Geophys. Res. Lett 37, L14107

González Hernández, I., Howe, R., Komm, R. W., \& Hill, F. 2010, ApJ (Letters), 713, L16

Hathaway, D. H. \& Rightmire, L. 2010, Science, 327, 1350

Howard, R. \& LaBonte, B. J. 1980, ApJ (Letters), 239, L33

Howe, R., Christensen-Dalsgaard, J., Hill, F., Komm, R. W., Larsen, R. M., Schou, J., Thompson, M. J., \& Toomre, J. 2000, ApJ (Letters), 533, L163

Howe, R., Christensen-Dalsgaard, J., Hill, F., Komm, R. W., Schou, J., \& Thompson, M. J. 2009, ApJ (Letters), 701, L87

Komm, R. W. 2007, AN, 328, 269

Komm, R. \& Hill, F. 2009, JGR, 114, A06105

Leibacher, J. W., Kholikov, S., \& Hill, F. 2010, BAAS, 42, 823

Mason, D. Komm, R. Hill, F. Howe, R. Haber, D. A., \& Hindman, B. W. 2006, ApJ, 645, 1543

Muñoz-Jaramillo, A., Nandy, D., \& Martens, P. C. H.. 2009, ApJ, 698, 461

Reinard, A. A., Henthorn, J. Komm, R., \& Hill, F. 2010, ApJ (Letters), 710, L121

Ulrich, R. K. \& Boyden, J. E. 2005, ApJ (Letters), 620, L123

Vorontsov, S. V., Christensen-Dalsgaard, J., Schou, J., Strakhov, V. N., \& Thompson, M. J. 2002, Science, 296, 101

Walén, C. 1944, Arkiv f. Mat., Astron. o. Fys., 30A (15), 1

\section{Discussion}

BRANDENBURG: The torsional oscillation pattern from helioseismology assumes northsouth symmetry. How does this compare with the observed torsional oscillation at the surface? 
HiLl: The surface measurements show a difference between the zonal flow in the north and south hemispheres. The measurements from global helioseismology are necessarily symmetric across the equator due to the spherical harmonic decomposition of the data. However, we have also determined the torsional oscillation pattern from the ring diagram method of local helioseismology and these results show a north-south asymmetric pattern.

TOOMRE: What are the areas of emphasis for GONG science, now in its mature phase, for the coming decade?

HILL: The helioseismic section of GONG will focus on observations of the sub-surface flows and their relationship to the solar cycle and the surface activity. Since the solar cycle occurs on decadal time scales, we need continual, consistent, and long-term measurements of the interior dynamics to understand it. GONG will also develop space weather forecast tools based on helioseismology, investigate the relationship of the flows and the direction of the magnetic field using vector magnetograms, and seek to detect sub-surface magnetic fields.

Thompson: You showed the positive correlation between inferred subsurface vorticity and surface magnetic field. I still have a nagging concern that these measurements are made in regions of strong magnetic field where we don't fully understand the forward problem of wave propagation. Do you agree that there may be systematic errors and the vorticity could be an artifact?

HiLl: It seems to me that the ring-diagram analysis is less susceptible to magnetic contamination in comparison with the time-distance approach. The major concern for the rings is the suppression of the amplitude of the oscillations in active regions. We can test this by artificially suppressing the $p$-mode amplitudes in quiet-sun control areas, and reanalyzing the data to see if the vorticity pattern appears. 